\title{
UPTAKE AND METABOLISM OF TRICHLOROETHYLENE
}

\author{
JAMEs ParkhouSE, M.A., M.D., F.F.A.R.C.S., D.A. ${ }^{\circ}$
}

IN STUDYING the pharmacokinetics of inhalation anaesthesia it is usual to begin with the working assumption that if a certain amount of anaesthetic is given through the lungs the same amount will eventually leave the body, through the lungs, after the administration is over. We recognize that there are small losses through the skin, the wound, and the urine, but the general assumption has served for most practical purposes. Trichloroethylene has long been regarded as an exception, since metabolism occurs to an extent variously estimated in various circumstances. More recent evidence suggests that other inhalation anaesthetics, for instance halothane, are also metabolized to some extent.

Over the years a good deal of attention has been paid to trichloroethylene, not only because of its clinical use but also because of the potential toxicity of the drug and its metabolites in industry. Most studies relate to the breathing of very low concentrations over long periods of time, as might occur in a factory; Bartoniček, ${ }^{1}$ for example, estimated that when human volunteers inhaled trichloroethylene for five hours, 51 to 64 per cent was retained, but in these studies the inhaled concentration was $1042 \mu \mathrm{g} / \mathrm{L}$, which represents approximately 0.19 per cent. To what extent the situation in clinical anaesthesia is comparable is not fully known.

\section{General Considerations}

If we measure how much trichloroethylene goes into the body through the lungs, and also measure how much comes out, then, by subtraction, we know how much has stayed behind. In the literature, the amount of anaesthetic inhaled minus the amount exhaled during the administration is described as the amount (or percentage) retained. Further excretion occurs through the lungs after the administration is over, and this is referred to as the amount (or percentage) recovered. If we take the amount inhaled and subtract the total amount exhaled, both during and after administration, the difference must be accounted for in terms of metabolism or non-pulmonary excretion. If we use $\mathbf{M}$ to denote amount, or mass, of trichloroethylene, we can write $\Sigma M I-\Sigma M E=\Sigma M M$; that is, the sum of all trichloroethylene inspired, minus the sum of all the trichloroethylene expired, equals the total amount metabolized, ignoring skin loss etc.

The word uptake has not been used here; we are speaking of intake minus output. Uptake is a more dynamic concept, appropriately applied to the state of affairs which obtains at some moment during the administration. If we attempt to analyse the situation at a given stage, we can ascertain how much anaesthetic has gone into the body up to that time and how much has already come out through the lungs. By subtraction we know how much is currently in the body. This anaesthetic must be either in the blood stream or in the tissues, ignoring the small

-Department of Anaesthetics, University of Manitoba. Present address: Faculty of Medicine, University of Sheffield, England. 
amount (in terms of mass) contained in the lung gases; otherwise it has already been metabolized. By measuring the blood concentration and making another subtraction, therefore, we should be able to estimate the amount currently either in the tissues or broken down. If we knew what metabolites to look for, and how to find them, a final subtraction should give us at least a rough indication of tissue uptake:

$$
\begin{gathered}
\text { THILENE IN - TRILENE OUT = TOTAL UPTAKE; } \\
\text { TOTAL UPTAKE - BLOOD ( AND LUNG GAS) CONTENT = TISSUE UPTAKE AND METABOLISM; } \\
\text { TISSUE UPTAKE AND METABOLISM - METABOLITE ACCUMULATION = TISSUE UPTAKE. }
\end{gathered}
$$

\section{The Fick Principle}

For a lung, or a piece of body tissue, the Fick principle tells us that if we know the concentration of a substance in the fluid entering the organ and the concentration in the fluid leaving, and if we also know the total quantity of fluid passing through the organ in unit time, we can derive the quantity extracted by the organ during that time. In the case of respiration, the fluid being air and the organ being lung, the appropriate statement would be $\dot{V}\left(\bar{F}_{I}-\bar{F}_{E}\right)=$ minute uptake through the lung, assuming a respiratory ratio of one.

For the circulation, the fluid being blood and the organ being all the body tissues perfused by this blood, the appropriate statement would be $\dot{\mathrm{Q}}(\overline{\mathrm{F}} \mathrm{a}-\overline{\mathrm{Fv}})=$ minute loss from the blood to the tissues.

Breathing and heartbeat are both discontinuous processes: the act of inspiration is a discrete event which occupies a certain quantum of time and carries a certain quantum of air into the lungs; the act of expiration is another discrete event which produces a quantum of air slightly less than the intake, in the usual case where $\mathbf{R}$ is less than one. Similarly, left ventricular systole is a discrete event which pumps a certain quantum of blood into the systemic circulation; right ventricular systole is a discrete event which pumps an equal quantum of blood into the pulmonary circulation, $R$ in this case normally being one. Thus we might regard the ultimate unit of respiratory activity as a breath and the ultimate unit of circulatory activity as a heartbeat. In practice, it is convenient to use minutes as time quanta, although the end of a minute may not exactly correspond with the end of a breath or the end of a heart beat. Then the relevant fluid volumes, for use with the Fick principle, are the minute volume for respiratory uptake and the cardiac output for circulatory distribution.

The amount of anaesthetic entering the lungs during a minute will be

$$
\dot{\mathrm{V}}_{\mathbf{I}} \overline{\mathrm{F}} \mathbf{I} \text {, }
$$

where $\bar{F}_{\text {I }}$ signifies the average inspired concentration of the anaesthetic during that minute. Likewise, the quantity of anaesthetic pumped into the systemic circulation by the left ventricle during a minute will be

$$
\dot{\mathrm{Q}} \overline{\mathrm{Fa}}{ }^{\circ}
$$

where $\overline{\mathrm{F}}$ a refers to the average arterial concentration during that minute.

"Note the distinction between, for example, $\overline{\mathrm{F}} \mathrm{v}$ and $\overline{\mathrm{F}} \overline{\mathrm{v}}$, the concentration being averaged in the first case and the blood being averaged (i.e. mixed) in the second. The average mixed venous blood concentration is $\overrightarrow{\mathrm{Fv}}$. 
The difference in average concentration between ingoing and outgoing fluid, divided by the number of time units involved, indicates the average rate of uptake during the time considered.

The difference in average concentrations multiplied by the total flow gives quantity of uptake; this is an actual quantity taken up during the time considered.

The sum of the quantities taken up during all time quanta gives total uptake to the time considered. This can be written

\section{$\Sigma \mathrm{Mu}$}

or, if we think of our time quanta as infinitesimal,

$$
\int\left(F_{I}-F_{E}\right) d t, \text { or } \int\left(F_{a}-F_{\bar{v}}\right) d t
$$

as the case may be. In resorting to calculus, however, we should bear in mind that although we may construct a smooth curve by joining points which, for instance, define expired concentrations, there is no such curve in reality since expiration is a series of discrete events, each yielding a finitely different expired concentration.

\section{A Qualitative Example}

Suppose we set up the simplest form of controlled study - the administration of a known, constant concentration of trichloroethylene at a known constant minute volume. If we measure the expired concentration we shall find that it rises, with time, towards a plateau which will be appreciably below the inspired level. At equilibrium the difference between the inspired and expired concentrations is a measure of the metabolism of the drug plus the small non-respiratory losses which occur. Before equilibrium has been reached, the difference between inspired and expired concentrations is a measure of the rate of body uptake and metabolism. This rate will be high initially and will decline as equilibrium is approached. At equilibrium the rate of "uptake" will become constant but not zero, in the case of trichloroethylene, since it will include loss through metabolism. If the rate of metabolism were constantly to vary for some set of reasons, equilibrium could never be reached.

Applying the Fick principle to the respiratory system, we can derive the rate of uptake at any given time from the expression $F_{I} \dot{V}_{I}-\bar{F}_{E} \dot{V} E$, and we can sum the quantal uptakes for all time intervals to give a total body uptake, up to the time considered. For example, to the $i$ th minute of administration,

$$
\sum_{0}^{t} F_{I} \dot{V}_{I}-\sum_{0}^{t} F_{E} \dot{V}_{E}=\sum_{0}^{t} M U \text { (where } U=\text { total uptake) }
$$

Experimentally, the patient's expired air is very conveniently available to us. It does no harm to collect it all, analyse it, and measure its volume. If we do this, using a single container, the laws of physics will ensure that we obtain a true average expired concentration for the time period concerned. If we were merely interested in total uptake during the first thirty minutes, for example, we could collect all the expired gas during this time in a single large bag and analyse it, thus saving ourselves a great deal of calculation.

If we now look at arterial and mixed venous blood concentrations, these also 
will reach plateaus as equilibrium is attained, and they will remain different because of continuing metabolism. Their exact shape will depend on how much biotransformation of trichloroethylene takes place in the blood stream itself. In studying dog and rat tissues in vitro Butler ${ }^{2}$ found that chloral, which is probably the first breakdown product of trichloroethylene, was converted to trichloroethanol in all the tissues studied, including red blood cells, whereas trichloracetic acid was formed only in the liver and the kidney preparations. Clayton and I (1961) collected samples of arterial, mixed venous, hepatic venous, and portal venous blood during trichloroethylene administration to dogs, and from these data Mapleson deduced that metabolism of trichloroethylene certainly does not all occur in the liver, but that fairly close agreement with the experimental curves could be obtained by assuming breakdown in the blood stream.

Applying the Fick principle to the circulatory system, we can derive an expression for the amount of tissue uptake plus loss through metabolism - for example during the $i$ th minute:

$$
\dot{\mathrm{Q}}_{i}\left(\overline{\mathrm{F}} \mathrm{a}_{i}-\overline{\mathrm{Fv}}_{i}\right)=\mathrm{MTM}_{i}
$$

where $\mathrm{MTM}_{i}$ is the amount taken up by the tissues and metabolized during the $i$ th minute. Again, we can derive a rate of uptake from this and by summing the quanta for individual minutes we can obtain a total tissue uptake plus metabolism figure up to the $i$ th minute:

$$
\sum_{0}^{i} \operatorname{MTM}
$$

\section{The Body Defined}

By comparing inspired and expired gases we may draw conclusions concerning the amount of trichloroethylene which has entered "the body." In this calculation the dividing line between the body and the rest of the world is the point at which the streams of inspired and expired gas are separated. The measure of "uptake" thus achieved will include all the trichloroethylene in the respiratory passages, all the trichloroethylene in the lung gases, all the trichloroethylene currently in the blood stream, all the trichloroethylene taken up by the tissues, all the trichloroethylene disposed of by metabolism, and all the trichloroethylene excreted through the skin etc. This, then, is total uptake.

Calculations based on the arterio-venous difference indicate the amount of anaesthetic taken up by the tissues, lost through metabolism, and excreted through the skin; they do not include the amount remaining in the blood stream or the amount currently contained in the lung gases and airways. Haggard ${ }^{3}$ derived an uptake curve from the putative tissue tension multiplied by the body mass, and showed that this agreed with the total uptake measured from gas exchange. In this case the body mass included the blood volume; lung gases were ignored.

We cannot conveniently measure the blood content of anaesthetic at any moment in time, since this would require knowledge of the total blood volume and the average trichloroethylene concentration in all the blood in the body. Furthermore, mixed venous blood is not available to us in unlimited quantities, 
for experimental purposes, like expired air. We cannot innocuously obtain a true average mixed venous concentration over a given period of time by collecting all the blood ejected by the right ventricle.

The difference between total uptake and tissue uptake plus metabolism will clearly be largest at the beginning of the administration, when the arterial level is high and the mixed venous and tissue concentrations are still low. As administration proceeds towards equilibrium the difference will become small and constant, and will finally represent the amount of trichloroethylene in the blood at equilibrium. At this stage, the amount of trichloroethylene in the lung gas (expressed in terms of weight) will be an insignificantly small proportion of the total.

We can obtain some idea of the order of magnitude of difference from the data provided by Haggard ${ }^{3}$ for his Experiment One. This was an ether experiment on a dog weighing $10 \mathrm{~kg}$. After ten minutes of administration the arterial concentration was $0.6 \mathrm{gm} / \mathrm{L}$ and the mixed venous concentration was $0.24 \mathrm{gm} / \mathrm{L}$. We may assume, as Haggard did, that the total blood volume was about 10 per cent of the animal's body weight, and we may further assume that between 30 and 40 per cent of the total blood volume was "arterial" in nature. Haggard took the mixed venous concentration as an indication of mean tissue concentration and assumed a blood/tissue distribution coefficient of one. On this basis the amount of ether contained in the blood volume would have been about $0.38 \mathrm{gm}$, and the amount in the tissues about $2.4 \mathrm{gm}$. The mass of ether in the lung gases would have been of the order of $0.04 \mathrm{gm}$. Thus, approximately 15 per cent of the ether taken into the body during the first ten minutes would be contained in the lung gas and the circulating blood.

\section{The Influence of Metabolism}

The first metabolite to be identified was trichloracetic acid. ${ }^{4}$ Trichloracetic acid is excreted in the urine, and its production and excretion are slow in comparison to the time-course of clinical anaesthesia; Butler's data from the $\operatorname{dog}^{5}$ suggest that with a one-hour inhalation of trichloroethylene the maximum plasma level of trichloracetic acid is found one or two days later, and during the first 18 hours after exposure the rise in plasma level appeared very nearly linear. According to Butler, ${ }^{5}$ little if any of the trichloracetic acid derived from trichloroethylene is destroyed in the body.

Butler estimated the total production of trichloracetic acid from the plasma concentration and the urinary excretion. If this were the only metabolite, we should now have an indication of how to separate metabolism from tissue uptake:

MTM (from artereo-venous trichloroethylene difference) - MM (from trichloracetic acid production $)=\mathrm{Mr}$ (tissue uptake).

As equilibrium approaches, the tissue uptake of trichloroethylene will come closer and closer to zero, so that the curve representing tissue uptake plus metabolism will eventually merge with the curve representing metabolism.

However, there are other metabolites to be considered and, in practice, the grouping of all tissues together is too much of an oversimplification. But the general relationships are not without importance: for any given administration 
of trichloroethylene the proportion of the drug which is excreted through the lungs and the proportion which is metabolized might be expected to depend on the relative rates of these two processes. This could have a bearing on the clinically observed after-effects of administrations of different durations.

\section{EXCRETION}

When the administration of trichloroethylene is discontinued and the inspired concentration falls to zero, the quantal respiratory loss can be derived simply from measurement of the volume and trichloroethylene concentration of expired air. Rate of loss can be deduced from this, and so can total loss up to any time after the end of the administration.

Simultaneously, metabolism of the trichloroethylene remaining in the body will continue. The blood concentration of metabolites will continue to rise, and the urinary concentration of metabolites will become appreciable. All the time that the metabolite concentrations and output are continuing to rise, the blood trichloroethylene concentration will be falling, from the combined effects of pulmonary excretion and continuing breakdown.

Once the output of trichloroethylene from the lungs has fallen to negligible levels, we can say that "total body output" has virtually come to an end as far as the unmetabolized drug is concerned. By adding up all our measured quantal outputs we are then in a position to write the final statement with which we began:

$$
\Sigma M_{1}-\Sigma M E=\Sigma M M .
$$

If we can assume that the loss of unmetabolized trichloroethylene through the skin and in the urine is small, then the trichloroethylene taken in and not re-excreted must be accounted for by metabolism. If we continue to follow the progress of the metabolite that we have chosen as an example, trichloracetic acid, we can see how much of the retention of trichloroethylene in the body can be accounted for by this one metabolite. By a further subtraction, we should know roughly how much remains to be accounted for in terms of other metabolites, and if we set about measuring these, for example trichloroethanol and monochloracetic acid, we should ultimately be able to account for all the inhaled trichloroethylene.

This is a very general and rather superficial account of a very large problem. Experimentally, a great deal of work remains to be done before the various stages of the process can be elucidated.

\section{REFERENCES}

1. BARTONIČEK, V. Metabolism and Excretion of Trichloroethylene after Inhalation by Human Subjects. Brit. J. Indust. Med. 19: 134 (1962).

2. Butler, T. C. Reduction and Oxidation of Chloral Hydrate by Isolated Tissues in Vitro. J. Pharmacol. 95: 360 (1949).

3. HaGGARD, H. W. The Absorption and Elimination of Ethyl Ether. J. Biol. Chem. 59: 737, 753, $771(1924)$.

4. Barfett, H. M. \& Johnstone, J. H. The Fate of Trichloroethylene in the Organism. J. Biol. Chem. 127: 765 (1939).

5. Butren, T. C. Metabolic Transformations of Trichloroethylene. J. Pharmacol. 97: 84 (1949). 